\title{
MECHANICAL DESIGN OF A HIGH FIELD COMMON COIL MAGNET ${ }^{*}$
}

\author{
S. Caspi, ‥ Chow ${ }^{+}$, D. Dietderich, S. Gourlay, R. Gupta, A. McInturff, G. Millos, R. Scanlan, \\ Lawrence Berkeley National Laboratory, Berkeley, CA
}

\begin{abstract}
A common coil design for high field 2-in-1 accelerator magnets has been previously presented as a "conductorfriendly" option for high field magnets applicable for a Very Large Hadron Collider [1,2]. This paper presents the mechanical design for a 14 tesla 2-in-1 dipole based on the common coil design approach. The magnet will use a high current density $\mathrm{Nb}_{3} \mathrm{Sn}$ conductor. The design addresses mechanical issues particular to the common coil geometry: horizontal support against coil edges, vertical preload on coil faces, end loading and support, and coil stresses and strains. The magnet is the second in a series of racetrack coil magnets that will provide experimental verification of the common coil design approach.
\end{abstract}

\section{INTRODUCTION}

The superconducting magnet program at Lawrence Berkeley National Laboratory (LBNL) is focused on development of high field accelerator magnets using brittle superconductors. The current designs follow a "common coil" approach wherein a pair of racetrack coils is shared between two apertures to produce opposing fields in each aperture [1]. A 6 tesla magnet

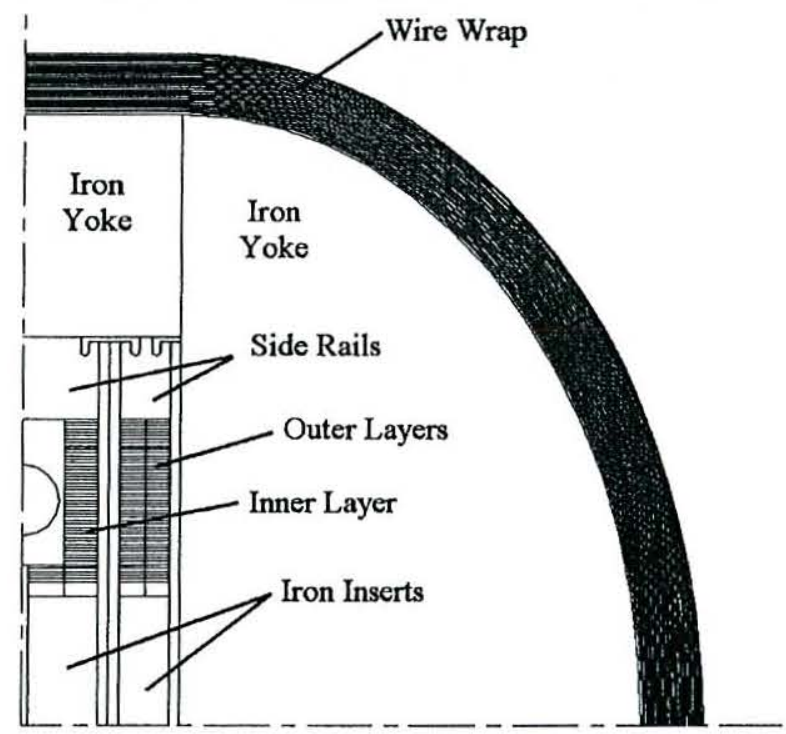

Figure 1: Basic components in magnet cross section (only one quadrant shown).

\footnotetext{
'Email: kpchow@lbl.gov

Work supported by the U.S. Department of Energy under contract No. DE-AD03-76SF00098.
}

using $\mathrm{Nb}_{3} \mathrm{Sn}$ conductor has been built and tested as a first step towards the ultimate goal of attaining $15 \mathrm{~T}$ accelerator quality dipoles $[3,4]$. This paper describes the mechanical design of a second magnet in the common coil series: a $14 \mathrm{~T} \mathrm{Nb}_{3} \mathrm{Sn}$ magnet of limited field quality (field quality will be addressed in the next magnet).

A preliminary design has been previously described elsewhere [2]. The design has since been refined to allow a modular and flexible R\&D program for systematic testing of different issues related to high field magnets that use brittle superconductor. The design also addresses several mechanical issues particular to a high field common coil geometry: horizontal support on coil edges, vertical preload on coil faces, end support, and coil stresses and strains. Figure 1 shows the basic components in a cross section of the magnet straight section.

\section{MAGNETIC DESIGN}

The primary purpose of this magnet is to demonstrate the viability of high field dipoles based on the common coil geometry in a mechanical structure suitable for high field accelerator magnets. As such, field quality issues will not be addressed in this magnet but will be fully addressed in subsequent common coil magnets [5]. Table 1 lists the major parameters of the present design.

Table 1: Major parameters of the present design.

\begin{tabular}{|l|c|}
\hline Bore diameter & $35 \mathrm{~mm}$ \\
\hline Main coil spacing (aperture) & $40 \mathrm{~mm}$ \\
\hline Computed quench field at 4.2 K & $14.3 \mathrm{~T}$ \\
\hline Peak field, inner layer & $15.1 \mathrm{~T}$ \\
\hline Peak field, outer layer & $10.3 \mathrm{~T}$ \\
\hline Quench current & $12.3 \mathrm{kA}$ \\
\hline Wire Non-Cu J. $\{4.2 \mathrm{~K}, 12 \mathrm{~T}\}$ & $2000 \mathrm{~A} / \mathrm{mm}^{2}$ \\
\hline Cu/Non-Cu ratio, inner \& outer & $0.7,1.7$ \\
\hline Strand diameter & $0.8 \mathrm{~mm}$ \\
\hline Number of strands, inner \& outer & 40,26 \\
\hline Cable width, inner \& outer & $17.2,11.3 \mathrm{~mm}$ \\
\hline Number of main coil layers & $1+3$ \\
\hline Straight section length, inner\&outer & $500,600 \mathrm{~mm}$ \\
\hline Number of turns (half magnet) & $5+48+48+48$ \\
\hline Height of each main coil layer & $80 \mathrm{~mm}$ \\
\hline Minimum coil bend radius & $70 \mathrm{~mm}$ \\
\hline Bore spacing & $220 \mathrm{~mm}$ \\
\hline Yoke outer height and width & $300 \mathrm{~mm}$ \\
\hline Total wire wind thickness & $30 \mathrm{~mm}$ \\
\hline
\end{tabular}




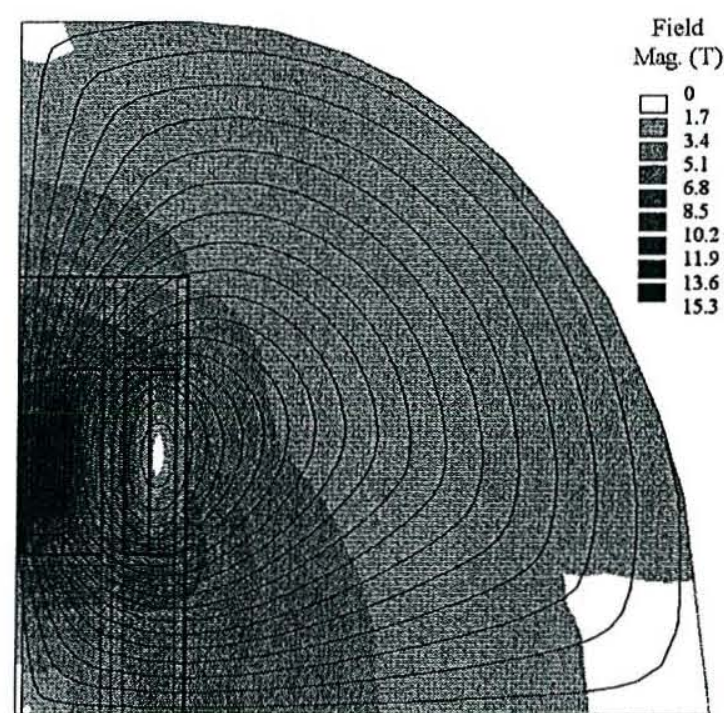

Figure 2: Field magnitude contours and flux lines in a quadrant of magnet cold mass (wire wind not shown).

This design uses a total of six main coil layers and two auxiliary coil layers (Fig. 1). Computed short sample (quench field) in the magnet bore is 14.3 tesla at $4.2 \mathrm{~K}$ (this field value uses the strand short sample and does not include any cable strain degradation). Figure 2 shows the field lines and field magnitude in a cross section of the current design.

\section{MECHANICAL DESIGN}

The mechanical design is governed by the need to support high Lorentz forces and manage coil stresses. The integrated horizontal Lorentz forces total 18.9 MN for the magnet, acting to push the windings apart. A rigid support structure is required to minimize coil displacements and maintain magnet integrity. Within the coil winding, Lorentz forces compress the winding and result in accumulated stresses approaching $150 \mathrm{MPa}$ (principal compressive stress). One method of reducing coil stresses being developed in a high field magnet design at Texas A\&M University is incorporation of rigid structural elements within the winding [6,7]. For the current $14 \mathrm{~T}$ design, no stress management is considered. The high coil stresses are not in areas of highest field and a previous high field magnet (D20) behaved well with similar levels of stress $[8,9]$. In addition to structural concerns, thermal conductance is an issue because adequate heat transfer is required for magnet thermal stability.

The current magnet design is based on design criteria for a flexible R\&D program. In contrast to accelerator quality production magnets, the magnet is designed to be modular; the magnet is separated into three types of components and each serves a dedicated function. The first component is the magnet core and is comprised of coil modules. The second component is the iron yoke, which serves as a flux return. The third component is an external wire wrap applied around the yoke to provide preload and support. Due to the modular nature of this design, the magnet can be reassembled with different coil modules for a flexible magnet system appropriate for conducting systematic R\&D parametric studies.

\subsection{Inner and Outer Coil Modules}

Vertical preload and support of magnet coil windings is provided completely by the coil module structure. The magnet core has three modules: one inner module sandwiched between two outer modules. Each outer module contains one double pancake winding, islands, end shoes, side rails, faceplates, and an iron insert. The inner module contains two pancake windings (each pancake has 48 turns for the main layer and 5 turns for the auxiliary layer), islands, end shoes, side rails, face plates, bore spacers, and iron inserts.

After epoxy impregnation of the $\mathrm{Nb}_{3} \mathrm{Sn}$ windings the outer coils are assembled into the coil module structure. The windings are preloaded in the direction normal to the cable faces and the module faceplates are welded to the side rails to maintain preload. For the outer coil modules, application of $150 \mathrm{MPa}$ of pressure on the cable faces yields $37 \mathrm{MPa}$ of preload after the faceplates are welded and the complete structure is cooled to $4 \mathrm{~K}$ (Fig. 3a, 3b). This preload prevents the coil inner turn from separating from the island when the magnet is energized. Magnet training is expected to be reduced by preventing energy release due to coil movement. Each outer module supports $450 \mathrm{kN}$ per meter of straight section length. The inner module is preloaded in a similar manner to the outer modules. For the inner module coil windings, $40 \mathrm{MPa}$ of vertical preload is required to prevent coil separation at the islands. The inner module supports $245 \mathrm{kN}$ per meter of straight section length.

Magnet ends are also supported within each coil module. Preload and support is provided through the module end shoes and reacted against the faceplates and side rails. The coil has a higher thermal contraction than iron inserts at the racetrack centers and the iron inserts exert an axial force against the winding ends when the magnet is cooled to $4 \mathrm{~K}$. In addition to this internal preload from the iron inserts, an external preload is also applied against the end shoes. A gap is designed into the iron inserts to balance the internal and external preload.

\subsection{External Support Structure}

The external support structure provides all of the horizontal preload and support for the magnet. The coil modules are assembled between a set of yokes comprised of two rectangular sections and two half-elliptical sections (Fig. 1). Thirty layers of $1 \mathrm{~mm}$ thick stainless steel wire will be wound on a bobbin around the yoke perimeter with a tension of $220 \mathrm{MPa}$ to preload the 


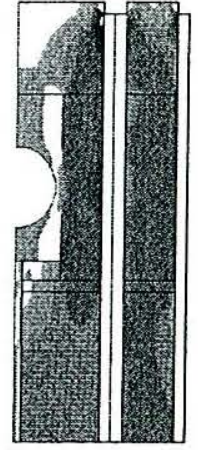

(a) Vertical Stres Room Temp

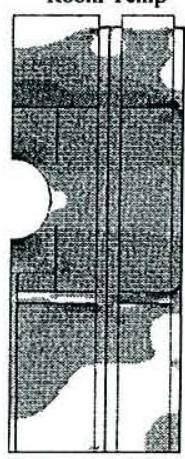

(d) Horizontal Str Room Temp

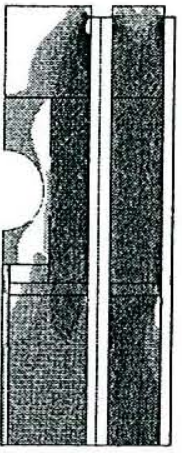

(b) Vertical Stress $4 \mathrm{~K}$ Temp

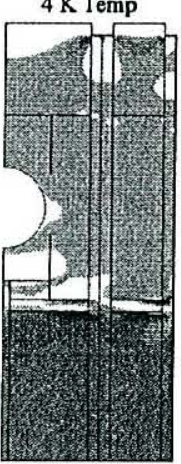

(e) Horizontal S $4 \mathrm{~K}$ Temp

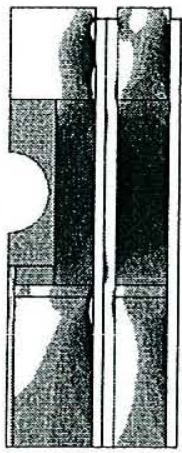

(c) Vertical Stres Energized

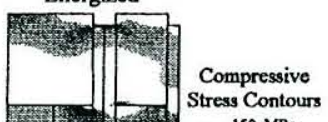
Stress Contour

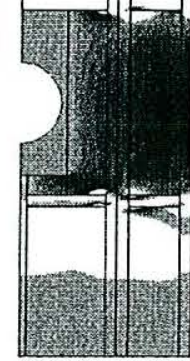

(f) Horizontal Stress Energized

Compressive ${ }_{133}^{150 \mathrm{MP}}$ 117
100
83.3 鯆 83.3 善 56.7 國 33.3 Stress Contours

Figure 3: Vertical and horizontal stresses in one quadrant of the coil module straight section (yokes not shown).

yokes and provide rigid support for horizontal Lorentz forces. After the last wire layer is applied, tension in the innermost wire layer decreases to $125 \mathrm{MPa}$. When the magnet is cooled to $4 \mathrm{~K}$ the wire tension increases to 300 $\mathrm{MPa}$ in the first layer and $400 \mathrm{MPa}$ in the last layer. This wire tension preloads the iron yokes in compression to minimize coil horizontal displacements when the magnet is energized. The total horizontal force of $18.9 \mathrm{MN}$ is supported by the preloaded yokes and wire winding.

The coil modules are designed to stack $0.05 \mathrm{~mm}$ wider than the rectangular yoke components. During the wire winding at room temperature, the coil modules are compressed horizontally to a maximum stress of about $30 \mathrm{MPa}$ (Fig. 3d). When cooled down to cryogenic temperatures, the coil modules contract more than the iron yoke components and the horizontal compressive stress on the coils decreases to less than $10 \mathrm{MPa}$ (Fig. $3 e$ ). (Correspondingly, the horizontal compression on the yokes increases as the magnet is cooled.) With less than $10 \mathrm{MPa}$ of horizontal preload, the coil surfaces on either side of the magnet bores separate from the bore spacer at low field and are essentially free at high fields.

When the magnet is energized, the stresses within the coil windings approach $150 \mathrm{MPa}$ in both the horizontal and vertical directions (Fig. 3c, 3f). However, the region of highest coil stress is not near the region of highest field. The region of highest horizontal stress coincides with the region of lowest magnetic field. The highest vertical stress exists in a region with a magnetic field magnitude of $9 \mathrm{~T}$. A previous epoxy impregnated $\mathrm{Nb}_{3} \mathrm{Sn}$ magnet behaved well with similar coil stress levels $[8,9]$ and we do not expect the high coil stresses to be a limiting factor. The epoxy impregnation increases the mean stiffness of the composite coil and provides support for the brittle superconductor. At field levels beyond 14 or $15 \mathrm{~T}$ the coil strength (epoxy impregnated) may become a critical issue and stress management may be necessary to prevent significant cable degradation.

\section{CONCLUSIONS}

The design of a high field 2-in-1 dipole based on a common coil design has been presented. The magnet is designed to provide a modular platform for flexible R\&D parametric studies of high field magnets using brittle superconductor. Coil modules may be replaced, added, or substituted to achieve new magnet configurations and improved field quality. An external wire winding structure that was successfully used in the past provides preload and supports the substantial horizontal Lorentz forces. The structure is also easily scaled for higher field magnets. Stresses within the coil winding when the magnet is energized approach critical levels; however, stress management has not yet proven necessary at $\sim 14 \mathrm{~T}$ field levels. Lastly, the common coil design uses flat racetrack coils with a large bend radius in the ends, enabling the future introduction of coils made with high temperature superconductors (HTS).

\section{REFERENCES}

[1] R. Gupta, "A Common Coil Design for High Field 2-in-1 Accelerator Magnets," Proceedings of the 1997 Particle Accelerator Conference, Vol. 3, pp. 3344-3346, May 1997.

[2] R. Gupta, et al., "A High Field Magnet Design for A Future Hadron Collider," Applied Superconductivity Conference, Palm Desert, CA, September 1998.

[3] K. Chow, et al., "Fabrication and Test Results of a Prototype, $\mathrm{Nb}_{3} \mathrm{Sn}$ Superconducting Racetrack Magnet," Applied Superconductivity Conference, Palm Desert, CA, September 1998.

[4] S.A. Gourlay, et al., "Fabrication and Test Results of a Prototype, $\mathrm{Nb}_{3} \mathrm{Sn}$ Superconducting Racetrack Dipole Magnet," 1999 Particle Accelerator Conference, New York, NY, March 1999.

[5] R. Gupta, "Common Coil Magnet System for VLHC," 1999 Particle Accelerator Conference, New York, NY, March 1999.

[6] N. Diaczenko, et al., "Stress Management in High Field Dipoles," Proceedings of the 1997 Particle Accelerator Conference, Vol. 3, pp. 3443-3445, May 1997.

[7] T. Elliot, et al., "16 Tesla $\mathrm{Nb}_{3} \mathrm{Sn}$ Dipole Development at Texas A\&M University," IEEE Transactions on Applied Superconductivity, Vol. 7, \#2, pp. 555-557, June 1997.

[8] A.D. McInturff, et al., "Test Results for a High Field (13 T) $\mathrm{Nb}_{3} \mathrm{Sn}$ Dipole," Proceedings of the 1997 Particle Accelerator Conference, Vol. 3, pp. 3212-3214, May 1997.

[9] S. Caspi, et al., "Development of High Field Dipole Magnets for Future Accelerators," Fifteenth International Conference on Magnet Technology, Beijing, China, October 1997. 